\title{
COMPUTATIONAL INVESTIGATION OF DISSIPATION AND REVERSIBILITY OF SPACE-CHARGE DRIVEN PROCESSES IN BEAMS
}

\author{
R. A. Kishek ${ }^{@}$, C. L. Bohn*, I. Haber ${ }^{\#}$, P. G. O’Shea, M. Reiser, and H. Kandrup ${ }^{\dagger}$, \\ Institute for Electronics and Applied Physics ${ }^{\ddagger}$, University of Maryland, College Park, MD 20742
}

\begin{abstract}
Collisionless charged particle beams are presumed to equilibrate via the long-range potential from the space charge. The exact mechanism for this equilibration, along with the question of macroscopic reversibility, has been uncertain, however. A number of computational approaches based on particle-in-cell (PIC) methods are presented which can facilitate the resolution of these questions. One such technique is the self-consistent tracking of individual particle orbits through the nonlinear potential formed by nonuniform charge density distributions. This orbit-tracking model differs from the particle-core model in that the sampled particles are systematically chosen from the actual particles in a fully self-consistent simulation. The results of this analysis are presented for a number of representative cases, and the implications of the study on equilibration mechanism are discussed.
\end{abstract}

\section{INTRODUCTION}

Charged particle beams with space charge are typically collisionless Hamiltonian systems where the detailed density distribution self-consistently governs the dynamics via Poisson's equation. The questions of equilibration, damping, and reversibility are of fundamental importance in determining beam properties. For example, equipartitioning of anisotropic beams involves nonlinear energy transfer and evolution towards an isotropic meta-equilibrium [1]. It is important for the accelerator designer to know whether the exchange of energy in this process is reversible. The same question arises when considering emittance growth due to misalignments or dispersion [2]. Reversibility implies the possibility of correction. Although extensive theory has been developed about thermodynamic equilibrium as it applies to beams [3], the absence of collisions necessitates the search for an alternative dissipation mechanism responsible for equilibration.

In this paper, we consider the possibility of one such dissipation mechanism: namely, phase mixing of globally chaotic particle orbits [4], which we call chaotic mixing. Chaotic orbits are characterized by sensitivity to initial conditions leading to exponential separation of nearby orbits. The process is therefore fundamentally irreversible. Further, globally chaotic orbits distribute themselves throughout their accessible phase space.

\footnotetext{
(a) $\overline{\text { ramiak@ebte.umd.edu }}$

* FNAL, Batavia, IL

\# Naval Research Lab, Washington, DC.

$\dagger$ U. of Florida, Gainesville, FL.

$\$$ http://www.ireap.umd.edu/umer/
}

If chaos turns out to be an important contributor to the dynamics of beams, then (i) the physics of rapid "relaxation processes" can be accessed and studied, and (ii) the accelerator designer will need to account for it.

We have developed computational tools, based on the particle-in-cell (PIC) code WARP [5], which we are using to investigate the behavior of interacting or noninteracting test particles affected by the self-consistent potential of the beam. We give examples involving both chaotic and regular orbits, and relate the associated dynamics to observable phenomena such as damping and equilibration.

\section{COMPUTATIONAL TOOLS}

For the simulations, we use the $2-1 / 2 \mathrm{D}$ version of the PIC code, WARP. Large numbers of particles are pushed in the self-consistent potential created by the space charge, as well as a predefined external field. For these preliminary runs we apply only a constant linear external focusing ("smooth approximation"), so any nonlinearity in the fields arises solely from the non-uniformities of the particle distribution. The degree of nonlinearity in the system can be manipulated by introducing more sophisticated geometries, such as a mismatch, a periodic lattice, anisotropy, altering the initial distribution, or any of host of complications.

Collections of test particles are also introduced at various locations of phase space. Each set of test particles ("ensemble") initially occupies a narrow portion of the 4$\mathrm{D}$ phase space, and is introduced in the code as a different species, albeit with the same charge and mass as the main beam, to ease in tracking it. The weight of macroparticles in each species can be adjusted at will. Thus we can make the test particles invisible to the main beam by setting the weight to zero; alternatively, we can give them any arbitrary non-zero weight, causing them to perturb the self-fields. The different ensembles are initialized to have a fraction of the size and velocity spread of the main beam, typically, 1/100. The moments of each species (the main beam being species 0 ) are calculated and saved periodically. Further, a small number of particles are selected at random from each species and their trajectories saved.

For the cases shown here, a constant focusing strength of $\kappa_{0}=15 \mathrm{~m}^{-2}$ is applied to a $10 \mathrm{kV}, 100 \mathrm{~mA}$ electron beam with an emittance of $50 \mathrm{~mm}-\mathrm{mr}$. This results in a beam radius of about $1 \mathrm{~cm}$, and a tune depression $\mathrm{k} / \mathrm{k}_{\mathrm{o}}$ of about 0.2 , making the beam highly space-charge- 
dominated. Typical numerical parameters are: $256 \times 256$ cells across the $5 \mathrm{~cm}$ diameter of the beam pipe; 4 million particles in the main beam (or 121 particles/cell); 20,000 particles in each test ensemble; and a step size of $2 \mathrm{~cm}$, significantly smaller than any of the characteristic wavelengths of the system $\left(\lambda_{\mathrm{p}}=1.14 \mathrm{~m} ; \lambda_{\beta \mathrm{o}}=1.63 \mathrm{~m}\right.$; $\lambda_{\beta}=12.6 \mathrm{~m}$ ). The initial distribution used in this case is a semi-Gaussian, although others will be attempted.

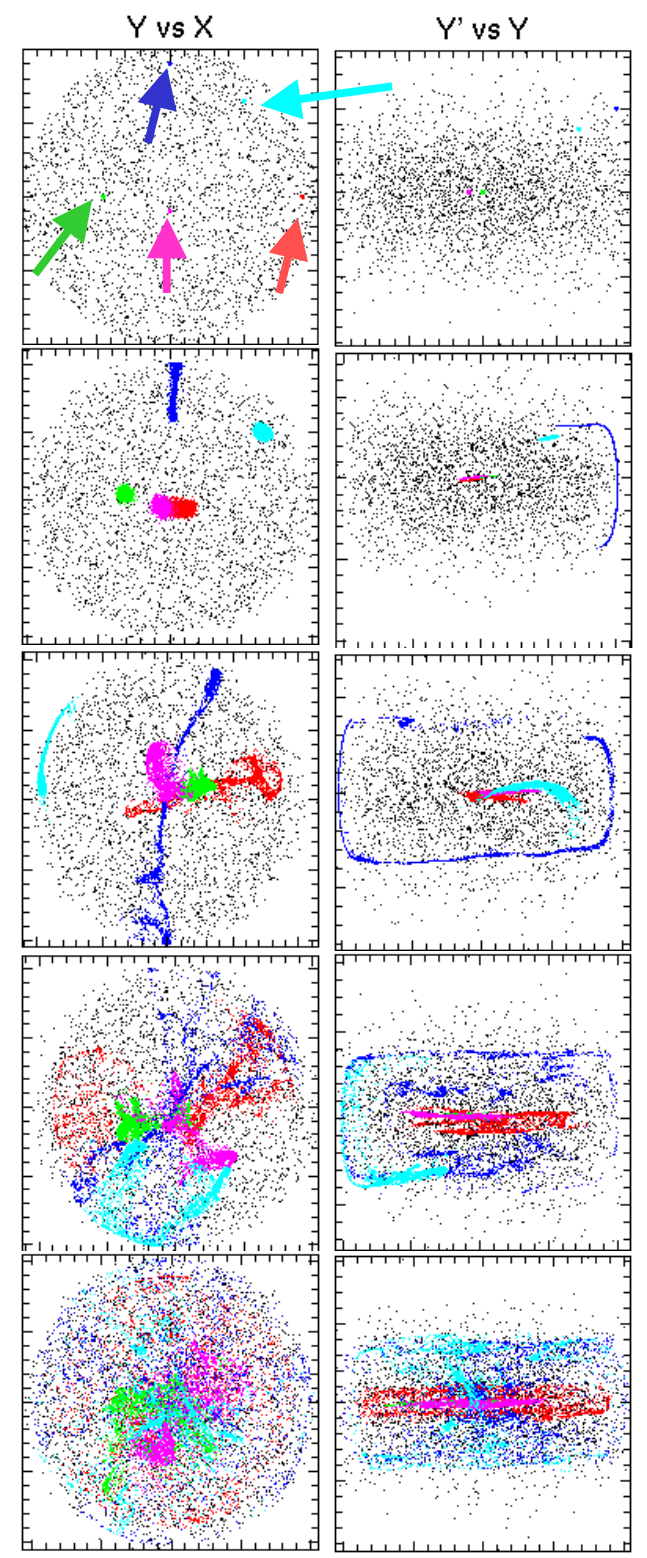

Fig. 1: Sequence of particle plots with colored ensembles, taken at distances of $0.0,11.52,51.84,100.8,169.9 \mathrm{~m}$ (top to bottom). Left is an $\mathrm{x}-\mathrm{y}$ projection, right is $\mathrm{y}-\mathrm{y}$ '.

\section{RESULTS}

Figure 1 demonstrates the growth of the size of each ensemble as a function of propagation distance, s. In this particular simulation, only the particles in the blue ensemble are interacting with the main beam (at full weight), while other ensembles are non-interacting. To ascertain whether the mixing is chaotic or not, we plot the moments of each ensemble on a semi-log scale, as shown in Fig. 2 for the "emittance" of the ensembles from the simulation of Fig. 1. It is apparent that for most ensembles, the exponential growth saturates at an early stage, before the particles fill even a fraction of the available phase space. Afterwards, the ensembles continue to grow in size, but not exponentially, indicating that the mixing mechanism is not globally chaotic. The small span of exponential growth at the beginning is an indication of local chaos, possibly arising from the particle noise. This is to be expected for a case like this, since the potential experienced by the particles is well behaved, and the only nonlinearities arise from relaxation of the particle distribution via small-amplitude space charge waves [6]. In this case, the blue ensemble has the fastest mixing rates, possibly because the blue ensemble acts as a perturbation to the potential in its vicinity.

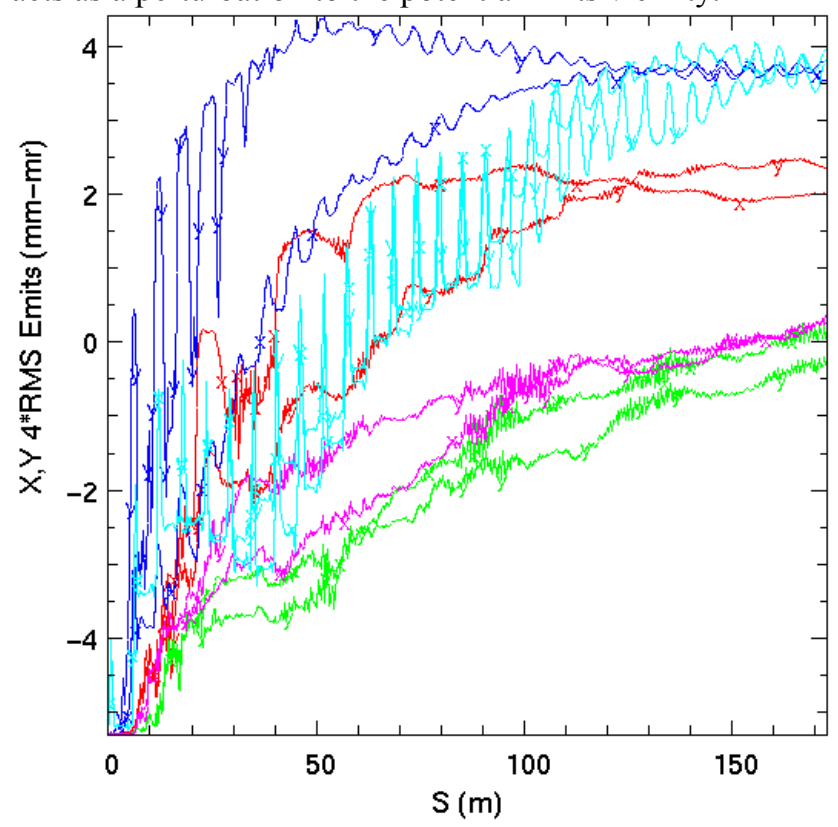

Fig.2: Evolution of natural logarithm of the "emittance" moment for ensembles in the isotropic beam of Fig. 1.

In order to observe more prominent mixing, we introduce an anisotropy by doubling the initial beam emittance in the $\mathrm{x}$ direction to $100 \mathrm{~mm}-\mathrm{mr}$ [we further adjust the focusing field and the beam size to maintain a matched beam]. This anisotropy in a symmetric focusing channel has been observed [1] to very quickly lead to equipartitioning by exchange of energy in the two transverse directions. Figure 3 illustrates the growth of the emittance moment of each ensemble for this anisotropic case. Contrasting this with the curves from Fig. 2, it is obvious that the mixing proceeds much faster for all the ensembles in the anisotropic case. We further observe that the majority of ensembles now continue the exponential growth until they fill a large fraction of the phase space before saturating. This is a good indicator of chaotic mixing. 


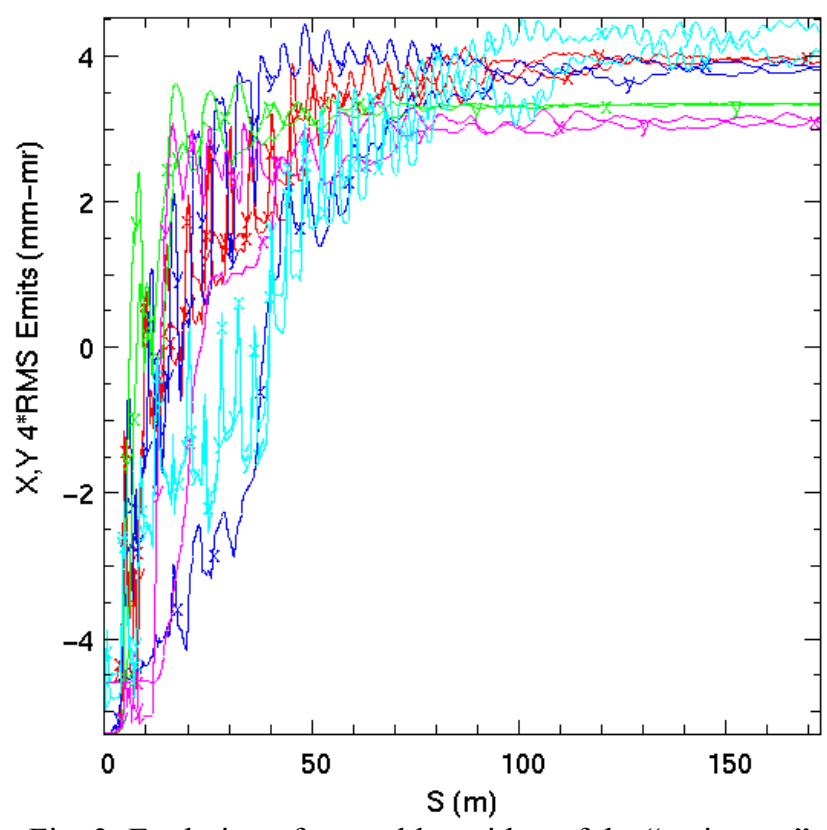

Fig. 3: Evolution of natural logarithm of the "emittance" moment for ensembles in the anisotropic beam.

The e-folding distance which can be measured from the figure is about $2 \mathrm{~m}$, roughly consistent with the scale over which the beam equipartitions (it takes about $5 \mathrm{~m}$ for the $\mathrm{x}$ and y emittances of the main beam to become equal). It therefore appears that the chaotic mixing is somehow associated with the equipartitioning of the anisotropic beam. The total distance it takes for the ensembles to fill the entire phase space (about $50 \mathrm{~m}$ ) is furthermore consistent with the damping scale of the remaining oscillations in the main beam emittance.

We can draw another comparison by examining the particle orbits in phase space. Fig. 4 contains such plots for two distinct ensembles. The one on the left represents the trajectories of 20 particles from the red ensemble in the simulation in Figs. 1 and 2, whereas the one on the right is from the green ensemble in the simulation of Fig. 3 . It is apparent that the orbits on the left are well behaved, whereas the ones on the right appear less regular and indicate possible chaotic behavior.

\section{CONCLUSIONS}

Preliminary results obtained so far indicate that there is a good possibility for chaotic mixing in beams. Much remains to be done to isolate real chaotic mixing from numerical effects and also to relate it to other phenomena such as instabilities. We wish to extend this investigation to more clearly delineate conditions under which chaotic mixing can take place. It is likewise conceivable that laboratory experiments in beams can be set up to study such physics with applications to other areas, such as galaxies [7], or large N-body systems of self-interacting particles in general.

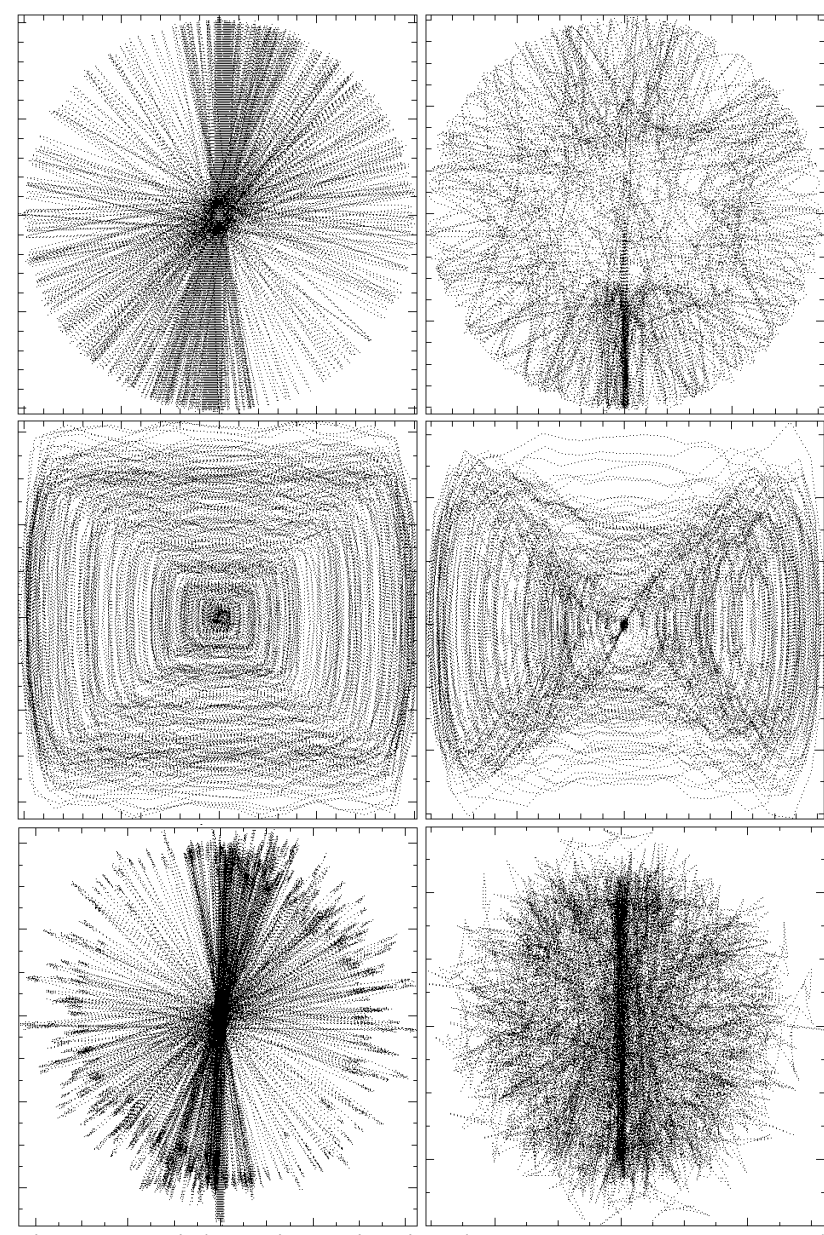

Fig. 4: Particle trajectories in phase space, $x-y, x-x$, and $x^{\prime}-y^{\prime}$ (top-bottom). 20 particles each from red ensemble of Figs. 1 and 2 (left); and green ensemble of Fig. 3 (right).

We thank Alex Friedman, Dave Grote, and Steve Lund for their excellent support of the WARP code. We further wish to thank S. Bernal and A. Valfells for valuable discussions. Research supported by US Dept. of Energy grant numbers DE-FG02-94ER40855 and DE-FG0292ER54178, and by Universities Research Association, Inc., under contract DE-AC02-76CH00300 with the U.S. Dept. of Energy.

[1] R. A. Kishek, P. G. O'Shea, and M. Reiser, Phys. Rev. Lett., 85 (21), 4514 (2000).

[2] M. Venturini, R.A. Kishek, and M. Reiser, PAC99, p. 3274 (1999).

[3] M. Reiser, Theory and Design of Charged particle Beams, (Wiley: 1994), chap. 5.

[4] C. L. Bohn, in The Physics of High Brightness Beams, (World Scientific, Singapore: 2000), p. 358.

[5] D. P. Grote, et. al., Fus. Eng. \& Des. 32-33, 193-200 (1996).

[6] S. Bernal, R. A. Kishek, M. Reiser, and I. Haber, Phys. Rev. Lett., 82, 4002 (1999).

[7] H. Kandrup, I. Pogorelov, and I. Sideris, Mon. Not. R. Astron. Soc., 319, 43-62 (2000). www.astro.ufl.edu/ siopis/papers 